\title{
Pyomyositis of the Piriformis Muscle-A Case of Piriformis Syndrome
}

\author{
S Shanmuga Jayanthan ${ }^{1} \quad$ S. Senthil Rajkumar ${ }^{1} \quad$ V. Senthil Kumar ${ }^{2} \quad$ M. Shalini ${ }^{3}$
}

${ }^{1}$ Department of Radiology and Imaging Sciences, Meenakshi Hospital, Tanjore, Tamil Nadu, India

2 Department of Orthopedics, Meenakshi Hospital, Tanjore, Tamil Nadu, India

${ }^{3}$ Department of Microbiology, Meenakshi Hospital, Tanjore, Tamil Nadu, India

Address for correspondence S. Shanmuga Jayanthan, MBBS, DNB, 27 , Third Cross Street, Subbaiya Pillai Nagar, Ammal Sathiram, Karaikal609604, Pondicherry, India (e-mail: jettan.32@gmail.com).

Indian J Radiol Imaging 2021;31:1023-1026.

\section{Introduction}

The piriformis syndrome is an uncommon form of sciatic nerve neuropathy secondary to nerve entrapment. ${ }^{1}$ This syndrome is characterized clinically by pain and paresthesia, in the ipsilateral gluteal region radiating into hip and posterior aspect of thigh, along the site of sciatic nerve innervation (sciatica). ${ }^{1}$ The most common cause for sciatica is lumbar disk herniation. ${ }^{2}$ However, there are other extraspinal causes for it and piriformis syndrome is one of them, which accounts for only $6 \%$ of cases. ${ }^{2}$ Pyomyositis of piriformis muscle is a rare cause for this syndrome. ${ }^{3}$ The most common causative organism is staphylococcus aureus. ${ }^{3}$ Both contrastenhanced computed tomography and magnetic resonance imaging (MRI) are useful in the diagnosis of piriformis abscess. ${ }^{4}$ However, MRI is more sensitive than CT at early stages, and hence, the former is the imaging modality of choice. ${ }^{4}$ Antibiotics are first line of treatment in patients with minimal inflammation and without abscess. ${ }^{4}$ However, patients with frank abscess require image-guided or surgical drainage, along with surgical decompression of sciatic nerve in the form of piriformis tendon tenotomy. ${ }^{4-6}$

\section{Clinical History}

A 41-year-old male patient presented with acute onset of pain in the left gluteal region and radiating into the posterior aspect of thigh. He gave history of fever for 1 week and laboratory parameters showed elevated white blood cell count $\left(16,608\right.$ cells $/ \mathrm{mm}^{3}$ ) with $80 \%$ neutrophils. There was no history of weight loss or antitubercular treatment. He is a known diabetic patient with elevated random blood sugars (246 mg/dL) and HbA1c (11\%).

On clinical examination, Pace's test (pain on resisted hip external rotation and abduction) and Freiberg's sign (pain with passive internal rotation of the hip) were positive, implicating piriformis syndrome. X-ray hip was unremarkable. published online

November 1, 2021
DOI https://doi.org/ 10.1055/s-0041-1739183. ISSN 0971-3026.

\footnotetext{
(C) 2021. Indian Radiological Association. All rights reserved. This is an open access article published by Thieme under the terms of the Creative Commons Attribution-NonDerivative-NonCommercial-License, permitting copying and reproduction so long as the original work is given appropriate credit. Contents may not be used for commercial purposes, or adapted, remixed, transformed or built upon. (https://creativecommons.org/ licenses/by-nc-nd/4.0/)

Thieme Medical and Scientific Publishers Pvt. Ltd., A-12, 2nd Floor, Sector 2, Noida-201301 UP, India
} 

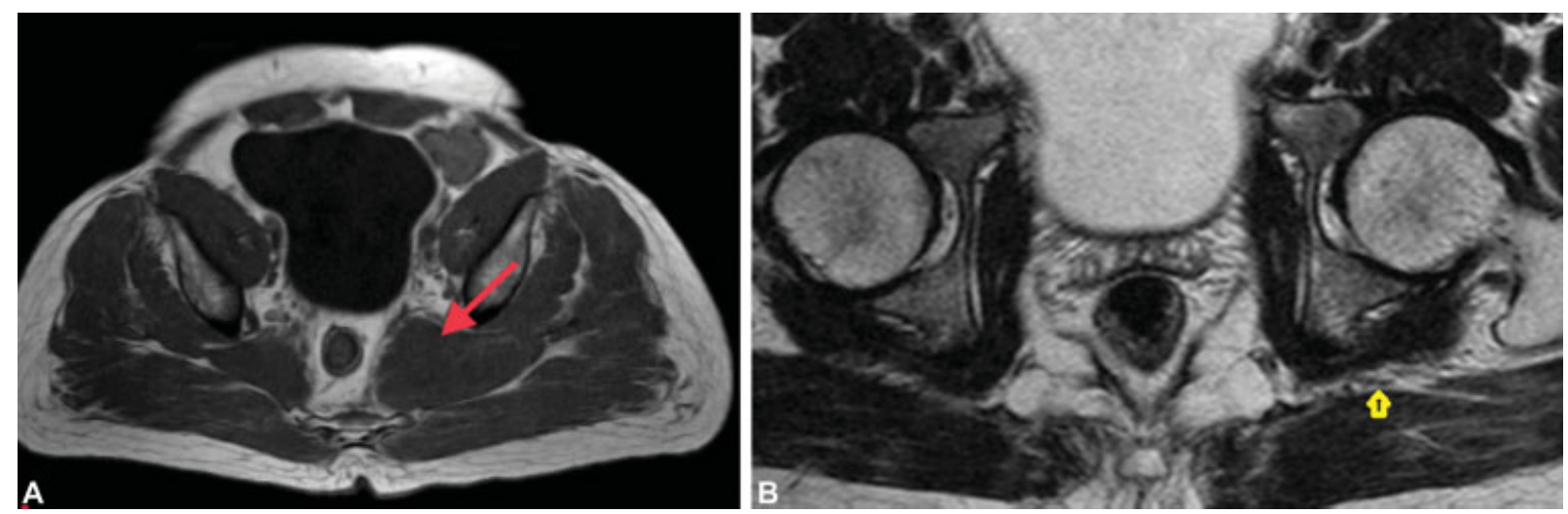

Fig. 1 (A) Axial T1-weighted image shows well-defined hypointense intramuscular collection in the left piriformis muscle. (B) Axial T2-weighted image shows the sciatic nerve below the level of greater sciatic foramen.

Initially, MRI of lumbar spine was advised, and MRI lumbar spine showed no significant abnormalities in disk. MRI-pelvis was, hence, considered with suspicion of extraspinal cause for sciatica. It showed a well-defined intramuscular $\mathrm{T} 1$ hypointense and $\mathrm{T} 2$ hyperintense collection $(3.8 \times 7.1 \times 2.5 \mathrm{~cm})(\mathrm{AP} \times \mathrm{ML} \times \mathrm{CC})$ in the left piriformis muscle with compression of an ipsilateral sciatic nerve ( - Figs. 1 and 2). STIR image showed diffuse muscular edema involving the left piriformis and glutei muscles (-Fig. 3). Postcontrast T1-weighted image showed well-defined, thick-walled collection with thin enhancing septa, suggestive of abscess (-Fig. 4). Bilateral sacroiliac joints were normal without

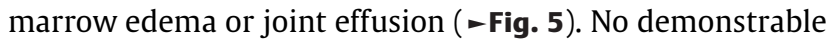
pathology in pelvic organs noted. Hence, the diagnosis of isolated left piriformis abscess causing piriformis syndrome was considered. Surgical drainage along with tenotomy of the piriformis tendon was performed ( - Fig. 6 ). Pus culture showed growth of staphylococcus aureus (-Fig. 7).

\section{Discussion}

Sciatica is a common neurological symptom, which is seen in approximately $40 \%$ of adults. ${ }^{2}$ It is characterized by radiating pain along the course of sciatic nerve, which is often secondary to disc degeneration that causes nerve root compression. ${ }^{2}$ However, there are other extraspinal causes for sciatica, which include, trauma, infections, sacroiliitis, tumors, and so on. ${ }^{2}$ Piriformis syndrome, an extraspinal

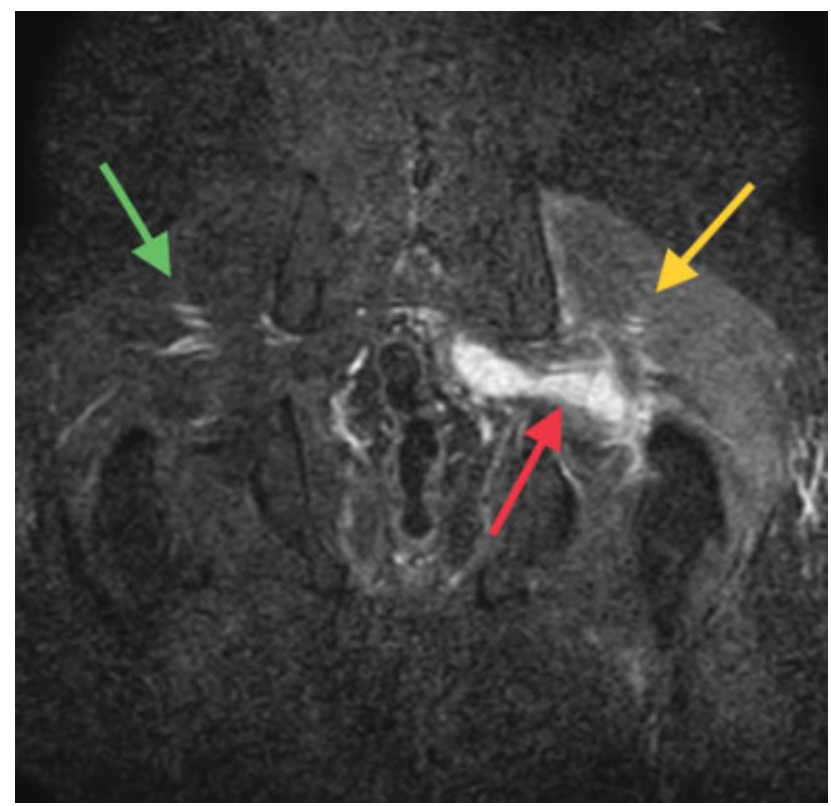

Fig. 3 Coronal STIR image shows the hyperintense intramuscular collection in left piriformis (red arrow), associated edema in left gluteal muscle (yellow arrow) compared with normal right gluteal muscle (green arrow).

cause for sciatica, is a form of sciatic nerve entrapment neuropathy at the level of greater sciatic notch. This occurs due to piriformis muscle abnormalities like hypertrophy, inflammation, or anatomical variations. ${ }^{1,2}$ This syndrome
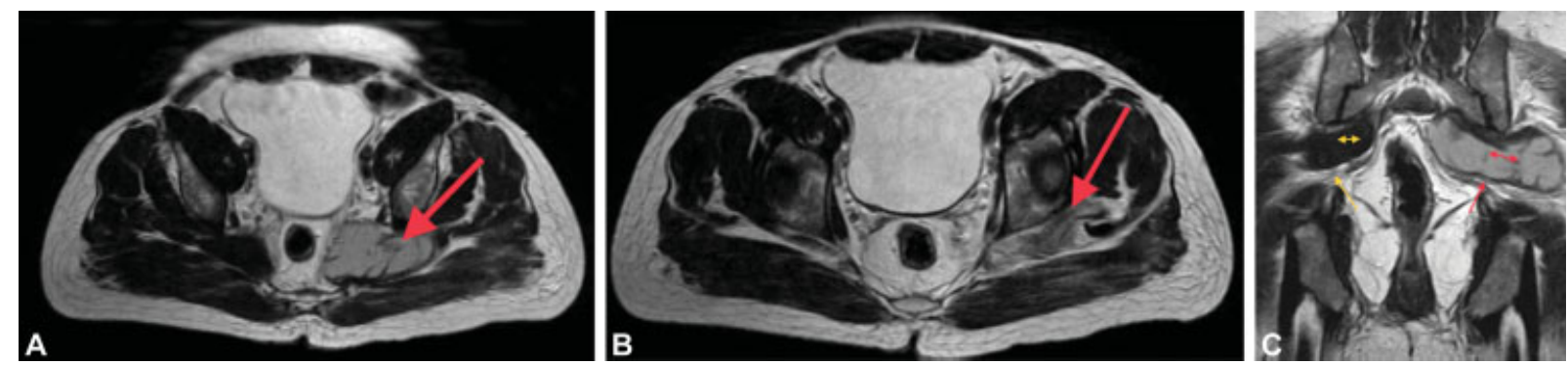

Fig. 2 (A) Axial T2-weighted image shows T2 hyperintense intramuscular collection with thin hypointense internal septations. (B) Axial T2weighted image shows the extent of the collection up to the left piriformis tendon. (C) Coronal T2-weighted image shows the normal relation of sciatic nerve (yellow arrow) to piriformis muscle (double-headed yellow arrow) on the right side and the impingement of sciatic nerve (red arrow) by the piriformis abscess (double-headed red arrow) on the left side. 



Fig. 4 (A) Axial T1-weighted postcontrast image shows peripheral enhancement of the T1 hypointense collection. There is also enhancement seen in the internal septations. (B) Coronal T1-weighted postcontrast image shows the T1 hypointense lesion with peripheral wall enhancement and enhancement of internal septations, suggestive of abscess.

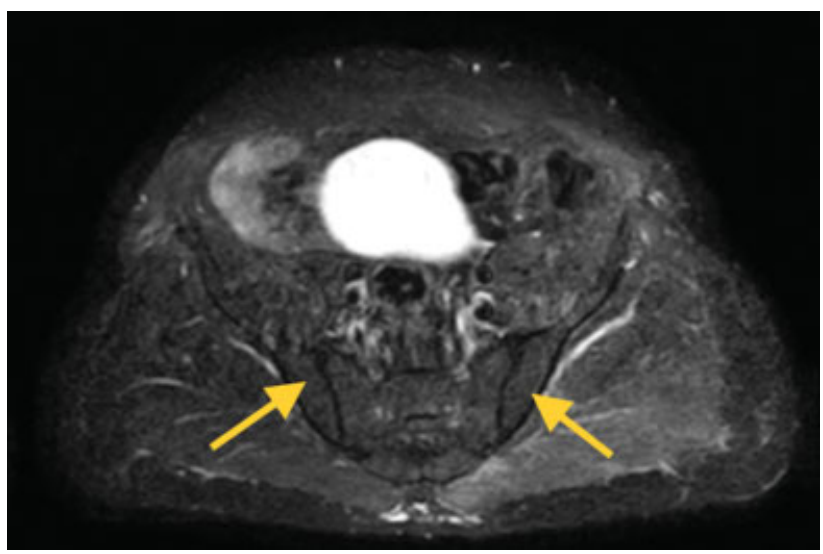

Fig. 5 Axial STIR image shows normal appearance of bilateral sacroiliac joints.

accounts for only approximately $6 \%$ of all cases of sciatic neuropathy. ${ }^{2}$

Pyomyositis of piriformis muscle can result in piriformis syndrome, and this is a very rare cause for piriformis syndrome. ${ }^{2,3}$ So far, only 23 cases of piriformis pyomyositis are reported in the literature. ${ }^{3}$ The risk factors include immunocompromised status, rheumatoid arthritis, diabetes, and prior parasitic or viral infections. ${ }^{4,5}$ Infections from spine and pelvis can also spread to the piriformis muscle through the endopelvic fascia. ${ }^{6}$ Two cases of piriformis pyomyositis were reported following obstetrical interventions. ${ }^{6}$

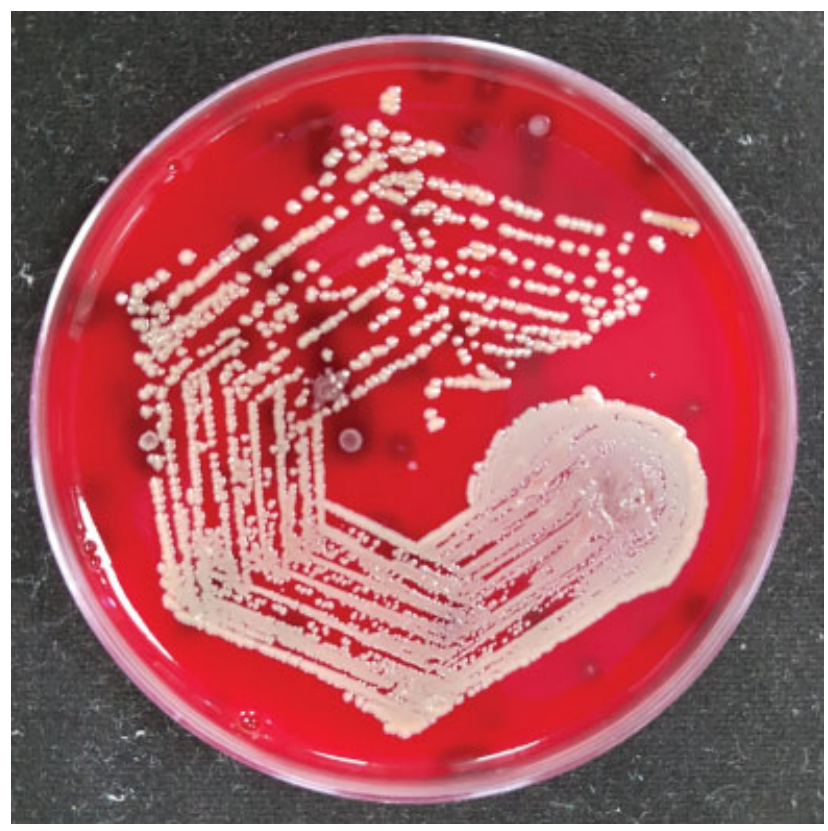

Fig. 7 Blood agar picture shows growth of Staphylococcus aureus colony.

Three phases of inflammation are seen. ${ }^{4}$ The first stage is nonpurulent, where there is only mild inflammation of muscle, the second stage is characterized by pus formation (purulent) within muscle, and third stage is identified by systemic toxicity. ${ }^{4}$ The most frequent causative organism is
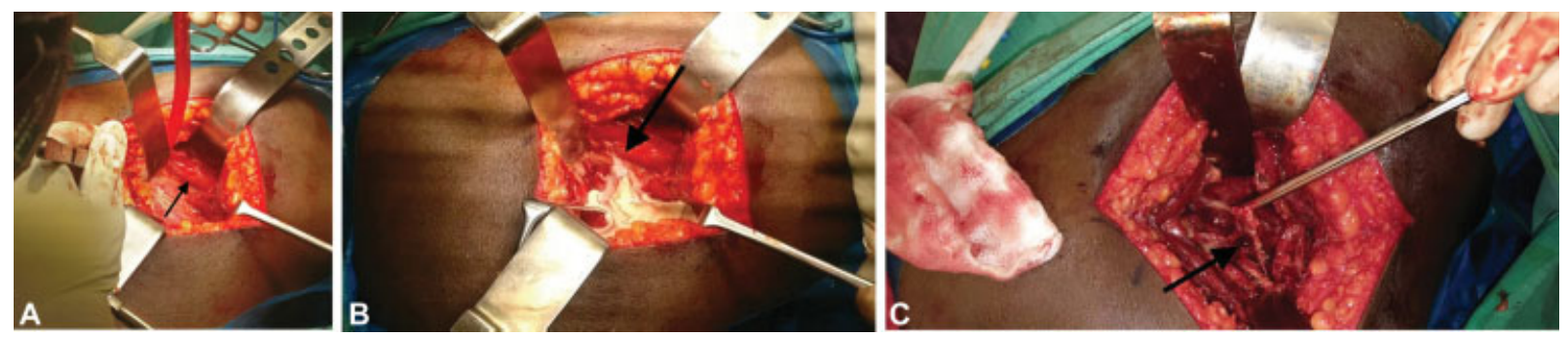

Fig. 6 (A) Intraoperative picture of enlarged piriformis muscle. (B) Intraoperative picture of surgical drainage of abscess. (C) Intraoperative picture of friable piriformis muscle tendon. 
Staphylococcus aureus, ${ }^{4,5}$ although the single case of brucella pyomyositis is described in literature by Kraniotis et al.

Clinical diagnosis is difficult as the muscle is deep from palpation and symptoms are related to sciatica. ${ }^{2,4}$ It is important to diagnose this condition at early presuppurative stage as the delay in diagnosis could result in increased morbidity and mortality. ${ }^{4}$ MRI plays an important role in the early diagnosis of this condition. ${ }^{1,4}$ If this condition is diagnosed at presuppurative stage, antibiotics alone would suffice and surgical drainage is reserved for cases with abscess and which do not respond to antibiotics.

Although contrast-enhanced CT can demonstrate intramuscular abscess with ring enhancement, ${ }^{2,5}$ MRI is the imaging modality of choice at early stages (presuppurative stage) due to its higher sensitivity. ${ }^{1}$ Findings in MRI at early stage include enlargement of muscle with T2 and STIR hyperintensity, without intramuscular fluid collection. Later stage, with abscess, is identified by intramuscular T1 hypointense and T2 hyperintense collection, which shows thick peripheral wall enhancement on postcontrast study. ${ }^{4}$ STIR hyperintensity can be seen within the muscle, suggestive of inflammatory edema. ${ }^{4}$ Anterior displacement of the sciatic nerve with T2 signal hyperintensity may also be observed. ${ }^{2}$ MRI can also exclude sacroiliac joint or pelvic pathology that can result in piriformis pyomyositis. ${ }^{4}$ We can also rule out disk abnormalities, which is the most common cause for sciatica. $^{2,4}$

Treatment for myositis is intravenous antibiotics at early stages of inflammation when there is no abscess. ${ }^{4}$ When there is abscess formation, CT-guided or surgical drainage of abscess, along with intravenous antibiotics, is the treatment of choice. ${ }^{4}$ It is important to be aware of this condition as early diagnosis can significantly reduce morbidity and mortality. $^{4}$

\section{Conclusion}

Although sciatica is commonly caused by disk pathology, it is important to get familiarized with extraspinal causes of sciatica. Piriformis syndrome is one among the cause for extra-spinal sciatica. Piriformis pyomyositis is a rare pathology causing piriformis syndrome but has significantly high mortality, if the diagnosis is delayed. MRI has an important role to play in the early diagnosis of this condition.

Financial Support and Sponsorship

None.

Conflict of Interest

None declared.

\section{References}

1 Lee EY, Margherita AJ, Gierada DS, Narra VR. MRI of piriformis syndrome. AJR Am J Roentgenol 2004;183(01):63-64

2 Ergun T, Lakadamyali H. CT and MRI in the evaluation of extraspinal sciatica. Br J Radiol 2010;83(993):791-803

3 Siddiq MAB, Rasker JJ. Piriformis pyomyositis, a cause of piriformis syndrome-a systematic search and review. Clin Rheumatol 2019;38(07):1811-1821

4 Kraniotis P, Marangos M, Lekkou A, Romanos O, Solomou E. Brucellosis presenting as piriformis myositis: a case report. J Med Case Reports 2011;5:125

5 Wong $\mathrm{CH}$, Choi SH, Wong KY. Piriformis pyomyositis: a report of three cases. J Orthop Surg (Hong Kong) 2008;16(03):389-391

6 Chong KW, Tay BK. Piriformis pyomyositis: a rare cause of sciatica. Singapore Med J 2004;45(05):229-231 